\title{
Using Functional Connectivity Analyses to Investigate the Bases of Autism Spectrum Disorders and Other Clinical Populations
}

\author{
Ryan A. Stevenson \\ Department of Hearing and Speech Sciences, Vanderbilt University Medical Center, Vanderbilt Brain Institute, and Vanderbilt University Kennedy Center, \\ Nashville, Tennessee 37205 \\ Review of Sepulcre et al.
}

A historically predominant view of the organization of the human brain has been a modular view where individual, isolated brain regions receive information, carry out a specific process, and pass that information on to other brain regions. The advent of functional magnetic resonance imaging (fMRI) initially did little to challenge this modular view. The first fMRI experiments identified particular brain regions active during simple tasks, such as finger movements (Bandettini et al., 1992), with little discussion of how these regions interact. Such studies have yielded a tremendous amount of information about the human brain, both in its healthy state and in a plethora of disease states. Despite the widespread success of this approach, it does not, perhaps, give the most accurate depiction of the brain as a whole.

Throughout the last two decades there has been a steady shift away from such a modular view of the brain toward a view better described as an integrated web of interactive and overlapping networks of

Received Sept. 22, 2012; revised 0ct. 5, 2012; accepted 0ct. 9, 2012.

I was funded through a grant from NIDCD, 1F32 DC011993, Multisensory Integration and Temporal Processing in ASD. My thanks to Mark Wallace.

The author declares no competing financial interests.

Correspondence should be addressed to Ryan Stevenson, 7110 MRB III,

BioSci Building, 465 21st Avenue South, Nashville, TN 37232. E-mail: ryan.andrew.stevenson@gmail.com.

DOI:10.1523/JNEUROSCI.4515-12.2012

Copyright $\odot 2012$ the authors $\quad 0270-6474 / 12 / 3217933-02 \$ 15.00 / 0$ brain regions. With such a viewpoint also comes the concept that studying brain regions in isolation cannot reveal how the brain functions as a system. Along with this paradigm shift (and in many cases driving the shift), analytical tools and concepts have been developed to describe and explore the functional brain as a network. One major step in this direction was the development of functional connectivity (Rogers et al., 2007). Functional connectivity correlates activity across brain regions in a cortical network. Thus, in addition to merely identifying and describing individual nodes in a network, functional connectivity can describe how these nodes are coupled across the brain, and how these couplings or changes in such couplings relate to particular processes or tasks. Further development of analytical techniques have provided means to describe these couplings in more detail, specifically revealing information on the directionality or causality of functional connections with tools including, but not limited to, Granger causality, structural equation modeling, and dynamic causal modeling (for review, see Bullmore and Sporns, 2009).

A second major step toward viewing the brain as a distributed network was the more rigorous application of graph theory to brain activity, again both theoretically and in the development of analytical tools (Bullmore and Sporns, 2009). Typical graph theoretical approaches treat individual voxels as nodes in a complex network and assess the structural and functional properties of how these nodes interact in a dynamical system. Most germane here are the following properties: (1) node degree, or the number of connections a node has within the network; (2) assortivity and clustering, which describe the topography of the connections between nodes, (3) hubs, which are central nodes in a network that serve as highly connected nodes that are vital to the integrity of the network; and (4) modularity, which describes the hierarchy of sub-networks within a larger complex network.

Measures derived from graph theory, much like basic measures of functional connectivity, commonly use measures that are symmetrical or undirected, providing no information on causality or direction of information flow through the network. With the introduction of stepwise functional connectivity (SFC) analysis in a recent issue of The Journal of Neuroscience, Sepulcre et al. (2012) have provided an analytical tool that has the ability to infer directional connectivity between nodes in a neural network. The initial steps of this analysis are similar to those of more canonical network analyses, establishing correlations between activity in nodes of the network and establishing which nodes are associated with other 
nodes, establishing correlations between activity in nodes of the network and determining which nodes are associated with other nodes, creating an undirected graph. The novel application used in SFC is what the authors refer to as a link step (Sepulcre et al., 2012, their Fig. 1). The number of link steps is similar to path length or the number of steps through associated nodes that it takes to connect a seed node to any other given node. Through comparisons of cortical maps derived from this linkstep measure, one can see the flow of information through cortex by comparing maps at one step, two steps, and so on until the map stabilizes at the cortical hubs (Sepulcre et al., 2012, their Fig. 2) In this way, Sepulcre et al. (2012) have provided a tool that not only adds exceptional ability to assess direction of information flow through a cortical network, but also allows visualization of how local network modules interact and merge to form a whole-brain network (Sepulcre et al. (2012), their Fig. 11).

While this analytical advance has clear and strong implications in furthering the basic understanding of neural networks, it also has the potential to be an important tool in clinical or applied research. Emerging evidence has identified a number of clinical disorders that are impacted by atypical connectivity in cortical networks, including the example that we will discuss here, autism spectrum disorders (ASD). The development of the SFC will allow researchers to directly test a range of clinical hypotheses about the neural underpinning of ASD. Experimental data from a typically developed population presented by Sepulcre et al. (2012) is relevant to the study of atypical connectivity in ASD. These data focused on the stepwise connectivity of visual, auditory, and somatosensory cortices (Sepulcre et al., 2012, their Figs. 3-5), specifically tracing the flow of information from primary sensory cortices through regions of multisensory integration and into neural hubs. These findings show that these three sensory modalities converge into similar brain regions, including occipitotemporal junction (OTJ), parietal operculum at the temporal parietal junction, superior parietal lobule, anterior insula, dorsal anterior cingulate (ACC), and prefrontal cortex.

These findings are applicable to ASD in a number of ways. First, dysfunction in visual, audition, and somatosensation modalities has been widely reported in autism, and the integration of information across sensory modalities is specifically impaired in ASD (for review, see Rogers and Ozonoff, 2005). In fact, several leading theories of autism, such as weak central coherence (Frith and Happé, 1994) and the temporal binding hypothesis (Brock et al., 2002) posit that the integration of information across brain regions is a core deficit in ASD. One hypothesis for these dysfunctions in sensory processing and sensory integration is that of degraded connectivity between brain regions and networks. For example, diffusion tensor imaging studies have shown reduced structural connectivity between sensory cortices and prefrontal regions and extrastriate visual regions and temporoparietal regions, as well as other regions identified as components of the multisensory network such as superior temporal sulcus, OTJ, and ACC (Barnea-Goraly et al., 2004; Shukla et al., 2011). Thus, measures of atypical connectivity that have been identified in ASD match quite well to the multisensory integration network mapped by Sepulcre et al. (2012). What these measurements of connectivity in ASD have not provided, however, is exactly how the flow of information in this sensory network is disrupted by the atypical connectivity patterns, a result of the undirected measures that have been used to identify the connections that are affected. SFC, however, provides a tool to do just that. The implications of moving from an undirected description of atypical connectivity to a directed description are quite important. For example, individual differences in how the flow of information is disrupted within the network may help explain the differences between the distinct endophenotypes seen in ASD.
Through their development of SFC, Sepulcre et al. (2012) have provided a new tool that allows for the directed analysis of complex neural networks, furthering the ability to study the brain as an integrated system. This analytical tool and the results reported describing the connectivity and flow of information from primary sensory cortices can be directly applied to clinical disorders associated with atypical connectivity, such as ASD. It should also be noted that, while the example of ASD serves to illustrate the possible translational aspects of SFC, ASD is joined by a multitude of other disorders that have been associated with atypical connectivity to which SFC could, and hopefully will, be applied.

\section{References}

Bandettini PA, Wong EC, Hinks RS, Tikofsky RS, Hyde JS (1992) Time course EPI of human brain function during task activation. Magn Reson Med 25:390-397. CrossRef Medline

Barnea-Goraly N, Kwon H, Menon V, Eliez S, Lotspeich L, Reiss AL (2004) White matter structure in autism: preliminary evidence from diffusion tensor imaging. Biol Psychiatry 55:323-326. CrossRef Medline

Brock J, Brown CC, Boucher J, Rippon G (2002) The temporal binding hypothesis of autism. Dev Psychopathol 14:209-224. CrossRef Medline

Bullmore E, Sporns O (2009) Complex brain networks: graph theoretical analysis of structural and functional systems. Nat Rev Neurosci 10:186-198. CrossRef Medline

Frith F, Happé U (1994) Autism: beyond "theory of mind.” Cognition 50:115-132. CrossRef

Rogers BP, Morgan VL, Newton AT, Gore JC (2007) Assessing functional connectivity in the human brain by fMRI. Magn Reson Imaging 25:1347-1357. CrossRef Medline

Rogers SJ, Ozonoff S (2005) What do we know about sensory dysfunction in autism? A critical review of the empirical evidence. J Child Psychol Psychiatry 46:1255-1268. CrossRef Medline

Sepulcre J, Sabuncu MR, Yeo TB, Liu H, Johnson KA (2012) Stepwwise connectivity of the modal cortex reveals the multimodal organization of the human brain. J Neurosci 32: 10649-10661. CrossRef Medline

Shukla DK, Keehn B, Müller RA (2011) Tractspecific analyses of diffusion tensor imaging show widespread white matter compromise in autism spectrum disorder. J Child Psychol Psychiatry 52:286-295. CrossRef Medline 